\title{
Does resuscitation in perforation-operation interval modify the mortality in duodenal perforations?
}

Vinod V. Prabhu, Sadanand G. Prasadi, Alka Gore ${ }^{1}$

Departments of Surgery and ${ }^{1} \mathrm{P}$ and $\mathrm{SM}$, Bharati Medical College, Sangli, Maharashtra,

India

Address for the Correspondence:

Dr. Vinod V. Prabhu, Shreyas, Behind Central Warehouse, Miraj - 416 410, Maharashtra, India. E-mail: cghert@yahoo.co.in

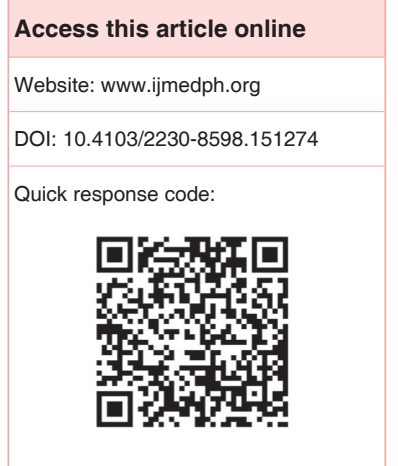

Objective: An attempt to analyze why in spite of prolonged perforation-operation (PO) interval mortality in patients of perforated duodenal ulcers is less in some patients and whether resuscitative measures in PO interval influences mortality particularly in a rural hospital. Materials and Methods: A retrospective analysis of 81 cases of perforated duodenal ulcers presenting in the surgical clinic in a rural medical college which is also a tertiary referral center. Resuscitation, if received in the PO interval was recorded, and a corrected score was calculated and compared statistically with the conventional Boey score. Results were analyzed using Microsoft Excel and SPSS 22 software. Results: Sample size of 81 patients had 70 referred and 11 direct patients. Six out of 11 direct patients succumbed $(P=0.545)$ and six out of 70 patients succumbed in the referred group ( $P=0.086$ ) suggesting that patients presenting directly to the tertiary center with a prolonged $\mathrm{PO}$ interval without any intervening resuscitation the prognosis is poor whereas a delayed $\mathrm{PO}$ with intervening medical intervention has a better prognosis. The PO interval was 23-168 h with a mean of 42.12 (standard deviation 26.86). Conclusion: In a rural referral center, the resuscitation done in the $\mathrm{PO}$ interval is significant as the prognosis is better in the group of patients resuscitated in the PO interval even if there is a delay in the surgical intervention whereas the mortality is higher in those group of patients who have come directly to the center and subjected to surgical procedure, but without any resuscitation in the PO interval. Hence, resuscitation in PO interval is paramount for a better prognosis.

Key words: Boey score, corrected Boey score, duodenal perforation, prognostic factors

\section{INTRODUCTION}

Perforated duodenal ulcer forms a substantial part of the patients attending surgical department. In a rural medical college majority of these patients are referred from a peripheral center or a private practitioner as a result of which the patients present late at the tertiary center that is, the perforationoperation (PO) interval is higher.

The conventional Boey's score does not take the preoperative resuscitation into consideration. But it has been observed that in spite of the delay, the mortality of these patients is surprisingly lower.

On evaluating the causes, it was found that these patients have been resuscitated at the peripheral center by inserting Ryles tube, intravenous (I.V) fluids, and injectable antibiotics.

The presence of various predictive scores available in the literature indicates a lack of consensus about prognostication. ${ }^{[1,2]}$ This is compounded by various hurdles faced by institutes like ours that caters to a predominantly rural population in a geography where access to the tertiary center is difficult due to socioeconomic backwardness. Here, the delay to access definitive surgical treatment is inevitable due to a lack of transportation and nonavailability of surgical expertise.

Patients with perforated duodenal ulcer often present with acute, severe illness that carries a high morbidity and mortality. Mortality varies from 3\% to $40 \%$ and several prognostic scoring systems have been suggested. ${ }^{[3]}$ The aim of this study was to evaluate the effects of resuscitation in the PO interval and to incorporate the same in the Boey's score. 
The preliminary resuscitation in the PO interval affects the mortality and hence have been incorporated in the Boey's scoring system so as to get a corrected score which predicts mortality, clinically, fairly accurately without taking any laboratory parameters into consideration. This, however does not obviate the prompt surgical intervention required in such patients nor is the delay justified. Delay in surgery is one of the most significant causes of mortality. ${ }^{[4-6]}$ However, if the delay is unavoidable or forced by socioeconomic reasons and there is difficulty in availing specialized surgical facilities then resuscitative measures in this PO interval will certainly decrease mortality.

\section{MATERIALS AND METHODS}

The retrospective study involved 81 consecutive duodenal ulcer perforation patients attending the outpatient department and casualty of our medical college hospital. All the patients were operated by laparotomy with Graham's patch.

The patients were divided into two groups, viz. Referred and Direct based on the referral letters.

Direct patients were those patients coming to us without any preliminary resuscitation or reference. Referred patients were those who were referred from a peripheral medical practitioner. The resuscitative measures received if any by the referred patients at the peripheral center were recorded, that is, I.V fluids received, Ryles tube in place and antibiotics received. The patients were allotted a score [Table 1], where treatment received is in the negative alongside the conventional Boey's score. The maximum score possible was +3 and the minimum -3 . A negative score was used as it counters the causes responsible for mortality.

Corrected score may be in negative depending upon the original score. A corrected Boey score was recorded for each patient to be evaluated against existing references in literature. Major illness included Ischemic heart disease (IHD), renal failure, pneumonitis, thrombocytopenia $<1 \times 10^{5}$, anemia $(\mathrm{Hb}<5 \mathrm{~g}$ $\%)$, and jaundice.

Other hollow viscus perforation, pediatric, and malignant perforations were excluded. Patients with incomplete data records were also excluded to avoid interpretational errors.

On discharge, the duration of stay of each patient, complications and cause of death were recorded. These data were analyzed using appropriate statistical tests.
Trial version of SPSS-22 (IBM, Bangalore), MS-Excel (Microsoft) was used for statistical analysis to use percentage, Z-test, Chi-square test for data analysis.

\section{Observation}

The mean age for the 81 patients was $52.62 \pm 15.823$ years (range, 17-90 years), $P=0.137$ suggesting age independence. A male predominance (70 male vs. 11 female) was observed $(P=0.293)$. Twenty-six patients $(32 \%)$ had an underlying medical illness $(P=0.250)$. Using Chi-square test, the $P$ values suggest that the score is independent of age, sex, and major illness. The delay between the start of symptoms and presentation to hospital that is, PO interval was 23-168 h with a mean of 42.12 (standard deviation 26.86). Twenty-six patients $(n=26)$ presented $<24 \mathrm{~h}$ after complaining of abdominal pain and $55(n=55)>24$ h of start of symptoms. There were 70 referred patients and 11 direct patients. Here, direct patients implied that these patients attended the referral center without being referred and not subjected to resuscitative measures whereas referred patients were those who were referred from peripheral centers and practitioners.

On comparing the mortality rates in referred and direct patients the proportion of deaths in direct patients is more $(Z=6.133$; $P<0.01)$ with a high statistical significance, implying that patients who have received resuscitation before admission for surgery have a better chance of survival. It is observed that after application of corrected criteria the percentage of mortality in score 0 and score 1 is zero and 20 patients have got a negative score, which is significant suggesting that after applying corrected score the percentage of survival has improved considerably whereas in score 2 and 3 the mortality is high. This indicates that even after applying corrected score if the score is high then the anticipated mortality increases [Table 2].

The patients received different types of resuscitation prior to institutional admission. It is observed that there were eight patients in the referred group who had received no resuscitation in the PO interval [Table 3] out of which six patients succumbed. Two patients survived because their referral to the tertiary center was $<24 \mathrm{~h}$ after the onset of symptoms whereas the presentation was $>24 \mathrm{~h}$ for the patients who succumbed strengthening the fact that a delay with no resuscitation in the PO interval is fatal.

It was observed that patients who had received resuscitation in the form of I.V fluids, Ryles tube, antibiotics either in isolation or various permutations with I.V fluids as a consistent feature had no mortality, whereas those patients who were referred without any

\begin{tabular}{|c|c|c|c|c|}
\hline Conventional Boeys score-B1 & Points & $\begin{array}{l}\text { Boeys score at } \\
\text { referral center-B2 }\end{array}$ & Points & $\begin{array}{c}\text { Corrected Boeys } \\
\text { score-B1 + B2 }\end{array}$ \\
\hline Presentation $>24 \mathrm{~h}$ & +1 & I.V fluid replacement & -1 & \\
\hline Systolic blood pressure $<100 \mathrm{~mm} \mathrm{Hg}$ & +1 & Ryles tube in place & -1 & \\
\hline Major illness & +1 & Antibiotics given & -1 & \\
\hline None of the above & 0 & None of the above & 0 & \\
\hline
\end{tabular}

I.V = Intravenous 


\begin{tabular}{|c|c|c|c|c|c|c|c|}
\hline \multicolumn{4}{|c|}{ Boey score } & \multicolumn{4}{|c|}{ Corrected Boey score } \\
\hline Score & Patients & Deaths & Percentage & Score & Patients & Deaths & Percentage \\
\hline- & - & - & - & Negative $^{* \star}$ & $20^{*}$ & 0 & 0 \\
\hline 0 & 11 & 0 & 0 & 0 & $37^{*}$ & 0 & 0 \\
\hline 1 & 34 & 0 & 0 & 1 & $12^{*}$ & 0 & 0 \\
\hline 2 & 35 & 11 & 31.4 & 2 & $11^{* * *}$ & 11 & 100 \\
\hline 3 & 1 & 1 & 100 & 3 & $1^{* * *}$ & 1 & 100 \\
\hline
\end{tabular}

\begin{tabular}{lc} 
Table 3: Preadmission treatment modalities in \\
referred patients \\
\hline Preadmission treatment & Number of patients \\
\hline I.V fluids & 44 \\
I.V + Ryles tube & 09 \\
I.V + Ryles tube + antibiotics & 02 \\
Ryles tube + antibiotics & 00 \\
Antibiotics only & 01 \\
I.V + antibiotics & 06 \\
No treatment & 08 \\
\hline
\end{tabular}

I.V=Intravenous

resuscitation or those delayed admissions, directly had the highest mortality. On the comparison between this series and the reference series of Boey and Irvine which have a larger sample size there is a statistically significant difference in proportion of score 2 patients. As the values in score 0,1 are zero and score 3 is similar they cannot be statistically compared [Table 4].

However, it is observed that after application of corrected score significant number of patients have moved up the score ladder thus improving their chances of survival.

Although the mortality in direct and referred patients are 6 each, there is a highly significant difference in proportions of deaths, $(Z=6.133 ; P<0.01)$ [Table 5] suggesting a higher mortality in direct patients who have not received resuscitation in the PO interval. Further, if we analyze the mortality data the average delay in presentation to our center was $44 \mathrm{~h}$ in both direct and referred patients. The patients that succumbed, in both direct and referred cases, did not receive any resuscitation prior to admission which is quite a significant finding.

The comparison of mortality in various series also indicates that the corrected score gives a $0 \%$ mortality in the lower scores (negative, 0 and 1 score value) as compared to the other authors [Table 6]. The mortality in score 2 and 3 is high because these are the groups containing direct patients and patients referred, but untreated in the PO interval.

Thus, it is advisable to take into account the preoperative treatment received by the patient, before scoring them for prognostication as the mortality is influenced by it.

Use of corrected Boey's score fairly assesses the probability of mortality. The average duration of stay in referred patients was
12.12 days and direct patients was 14.3 days. The pre admission resuscitative measures also reflect in the duration of hospital stay.

The cause of death was mainly due to septic shock, IHD and Pneumonitis. There were six patients of surgical site infection (SSI) and one with abdominal wall cellulitis who survived with treatment. The patients who received prior antibiotics at the periphery had no SSI. The referred patients had received an average of three bottles of Ringers lactate (500 ml) I.V in $24 \mathrm{~h}$, along with antibiotics such as Ceftriaxone, Tinidazole, and Gentamycin.

\section{DISCUSSION}

Duodenal perforations are commonly encountered emergencies in surgical practice. Many scores such as the Boey's score, the Mannheim Peritonitis index score, American Society of Anesthesiologists score, APACHE II, Hacettepe score predict mortality in patients with peptic ulcer perforation and are used preoperatively in the scoring system. ${ }^{[7]}$ As we are aware, the commonest question posed to a surgeon by the relatives of a patient is what are the chances of survival of the patient, and it is in this context that the prognostication assumes significance. Boey's score, which is a score based on factors such as shock on admission, confounding medical illness, and duration of perforation, has been found to be a useful tool in predicting the outcome. ${ }^{[8]}$ In our opinion the Boey's score is a useful prognostic indicator, only at the first contact center because if used at the tertiary center it would be erroneous as it would not take the preoperative resuscitative treatment into account.

Improving timely access to a good medical center, lessens the delay and may improve the outcome of patients undergoing surgery for perforated peptic ulcer. ${ }^{[9]}$

Co-existing medical illness, preoperative shock, compromised renal function and delay in treatment are significant risk factors that increase mortality in patients with perforated peptic ulcers. These factors could serve as a guide to opine about the risk to life and to improve the outcome in patients with perforated peptic ulcer. As is known, perforation causes shock, infection, and septicemia, ${ }^{[10]}$ if there is a PO delay. Mortality could be reduced by preventing delay in diagnosis, treatment of any co-existing medical illness ${ }^{[11]}$ and providing appropriate nutrition and fluid support that includes I.V fluids, antibiotics, and Ryles tube insertion, that is, proper preoperative resuscitation which forms the mainstay of the treatment before surgery. ${ }^{[12]}$ 
The resuscitation received by the patients in the PO interval has a significant bearing on the outcome of surgery irrespective of age, comorbid factors, and sex particularly when patients are received at a center like ours from peripheral interior areas which lack proper operative facilities, scarce transport facilities, drought affected areas and last but not the least poor economic condition. ${ }^{[13]}$ Many authors have recognized the importance of PO interval and its effect on mortality, but we are of the opinion that the treatment received in this period has a significant effect on reducing the mortality.

The surprising decrease in mortality found in our series, in the resuscitated patients made us analyze the causes, which resulted in the finding that we had a large number of referred patients who, in spite of delayed presentation had a comparably low mortality resulting in the observation that these patients received some resuscitative intervention relevant to their disease that reduced their mortality. Hence, we came up with a scoring system that incorporated these points which could strengthen the existing Boey's score that could still be used as an effective tool in a peripheral center to predict mortality cost effectively. ${ }^{[14]}$ The present series reveals that the mortality is less when resuscitation is carried out in the PO interval. A high mortality was observed in nonresuscitated patients in both referred and direct groups thus signifying the importance of resuscitation in the PO interval.

This also suggests that proper resuscitation even at the tertiary level prior to subjecting the patients to surgery by properly hydrating the

\begin{tabular}{lcccc}
$\begin{array}{l}\text { Table 4: Statistical comparison of mortality } \\
\text { (for score 2) }\end{array}$ & \multicolumn{4}{c}{ Reference series } \\
\cline { 2 - 5 } Statistics & Boey & $\begin{array}{c}\text { Corrected } \\
\text { Boey }\end{array}$ & Boey & Irvine \\
\hline Sample size & $n=81$ & $n=81$ & $n=259$ & $n=265$ \\
$P$ & $P=0.314$ & $P=1$ & $P=0.45$ & $P=0.55$ \\
$Z$ & $9.031^{*}$ & $8.627^{* *}$ & $7.324^{* * *}$ & \\
Standard error & $0.07459^{*}$ & $0.66281^{* *}$ & $0.06034^{* * *}$ & \\
of difference & & & &
\end{tabular}

*Comparing Boey and corrected Boey score, **Comparing corrected Boey and Boey reference series, $* * *$ Comparing corrected Boey and Irvine reference series

\begin{tabular}{lccc} 
Table 5: & Mortality & & \\
Group & Mortality & Total & Proportion \\
\hline Direct & 6 & 11 & 0.545 \\
Referred & 6 & 70 & 0.086 \\
\hline
\end{tabular}

$95 \%$ confidence interval for difference: $0.3169-0.6031 . Z=6.133 ; P<0.01$. There is statistically highly significant difference in proportions of deaths, between direct and referred patients patients so as to achieve an adequate urinary output and using better antibiotics may also bring down the mortality.

Does it mean that a policy of deliberate delay with adequate resuscitative measures is to be taken considering the evidence available in this study with the hope that the survival chances are more? Certainly not, as we are discussing only about the inevitable delays and not about the postoperative complications due to the delay, which contribute a great deal to mortality. It is prudent to understand that this observation could be taken as a positive suggestion to resuscitate the patient properly, even in a medical college before wheeling up the patient for surgery. Besides this, the PO interval can be utilized to insert a central venous line and improve renal function and in extreme cases insertion of abdominal drains under local anesthesia, which often takes a long period along with the change in duties of the on-call surgeons with a reluctance to operate on nonemergency days.

In a referral center, it is imperative to take the resuscitation received by the patients at the peripheral center into consideration in order to predict mortality. Taking this into account, we used the corrected Boey score. We found that a significant number of patients in the pre surgical resuscitated group moved up the scoring ladder, implying a good chance of survival. Although we had the basic laboratory parameters available to validate our observation we decided to use only the Boey's score along with the corrected Boey's score which would be simple to apply, obviate costly investigations and give a fair information about the mortality.

While there are a number of studies that include clinical evaluation, costly laboratory investigations ${ }^{[15]}$ we relied on the parameters laid down in Boey's score along with additions. The correction of fluid imbalance leads to an improvement in the physiology of patients, thereby improving tissue perfusion, preserving renal function, and maintaining patient nutrition. ${ }^{[16-19]}$ The Ryles tube helps in emptying the stomach and the regurgitant bile so as to prevent continuous soiling and minimizing the peritoneal reaction and secondary infection. The organisms that are frequently responsible for infection in gastroduodenal perforations are endogenous to the stomach and include aerobic enteric Gram-negative bacilli and oral anaerobes. ${ }^{[20]}$ The antibiotics take care of infection after the initial stage of the peritoneal reaction preventing sepsis which is a major cause of mortality. ${ }^{[21]}$

Thus, though literature mentions age, delay in surgery, sepsis, perfusion, comorbid conditions, and size of perforation as the major

\begin{tabular}{|c|c|c|c|c|c|}
\hline Score & $\begin{array}{c}\text { Boey }^{* *} \\
(n=259)(\%)\end{array}$ & $\begin{array}{c}\text { Lohsiriwat } \\
(n=152)(\%)\end{array}$ & $\begin{array}{c}\begin{array}{c}\text { Fung }^{* *} \\
(n=436)(\%)\end{array}\end{array}$ & $\begin{array}{c}\text { Chalya } \\
(n=84)(\%)\end{array}$ & $\begin{array}{c}\text { Corrected }{ }^{*} \text { Boey } \\
(n=81)(\%)\end{array}$ \\
\hline Negative score & - & - & - & - & 0 \\
\hline 0 & 0 & 1 & 1.5 & 0 & 0 \\
\hline 1 & 10 & 8 & 14.4 & 11.1 & 0 \\
\hline 2 & 45.5 & 33 & 32.1 & 33.3 & 100 \\
\hline 3 & 100 & 38 & 100 & 56.6 & 100 \\
\hline
\end{tabular}

*With lower scores in corrected Boey, the mortality is $0 \%$ as compared to other series, **Higher score has a higher mortality 
contributors to mortality, ${ }^{[22-24]}$ we observed that adequate perfusion, gastric decompression, and prevention of sepsis are paramount for patient survival ${ }^{[25]}$ particularly when a delay in reaching the tertiary care center is inevitable.

The mortality rate comparisons of various authors also point out to the fact that the corrected Boey score could be a reliable score [Table 6]. It is seen in this comparison of various series that survival rate prediction is better in score - ve., Score 0 and 1 in a corrected score which means that $85 \%$ of patient's relatives could be told that their patient could survive.

We feel that in a peripheral center receiving more referral patients, corrected Boey's score offers a good indicator of mortality that could be validated on prospective larger sample studies. The only shortcoming of this score according to us is that it does not prognosticate mortality due to postoperative complications.

\section{CONCLUSION}

Although age, PO interval, comorbid factors are important contributors to mortality we feel that the three most important factors used in pre surgical resuscitation that is, I.V fluids, Ryles tube, antibiotics used at the peripheral interior clinics or for that matter in the tertiary center in the PO interval prior to definitive surgery has a significant bearing on the mortality. In spite of the delay in definitive treatment, those patients who received resuscitative treatment in the PO interval had a better chance of survival than those who did not receive resuscitation.

However, as mentioned in the introduction, it does not in any way justify a delay in surgery with intervening resuscitation with the hope of reducing mortality. It could be used as a suggestion to resuscitate the patient properly prior to taking up the patient for surgery.

We feel that in a peripheral referral medical college hospital use of the corrected Boey's score could be used as an effective tool to prognosticate mortality with a need for prospective validation.

\section{REFERENCES}

1. Møller MH, Engebjerg MC, Adamsen S, Bendix J, Thomsen RW. The peptic ulcer perforation (PULP) score: A predictor of mortality following peptic ulcer perforation. A cohort study. Acta Anaesthesiol Scand 2012;56:655-62.

2. Buck DL, Vester-Andersen $\mathrm{M}$, Møller MH. Accuracy of clinical prediction rules in peptic ulcer perforation: An observational study. Scand J Gastroenterol 2012;47:28-35.

3. Thorsen K, Søreide JA, Søreide K. Scoring systems for outcome prediction in patients with perforated peptic ulcer. Scand J Trauma Resusc Emerg Med 2013;21:25.

4. Bas G, Eryilmaz R, Okan I, Sahin M. Risk factors of morbidity and mortality in patients with perforated peptic ulcer. Acta Chir Belg 2008;108:424-7.
5. Hermansson M, Staël von Holstein C, Zilling T. Surgical approach and prognostic factors after peptic ulcer perforation. Eur J Surg 1999;165: 566-72.

6. Kocer B, Surmeli S, Solak C, Unal B, Bozkurt B, Yildirim O, et al. Factors affecting mortality and morbidity in patients with peptic ulcer perforation. J Gastroenterol Hepatol 2007;22:565-70.

7. Mäkelä JT, Kiviniemi H, Ohtonen P, Laitinen SO. Factors that predict morbidity and mortality in patients with perforated peptic ulcers. Eur $\mathrm{J}$ Surg 2002;168:446-51.

8. Lohsiriwat V, Prapasrivorakul S, Lohsiriwat D. Perforated peptic ulcer: Clinical presentation, surgical outcomes, and the accuracy of the Boey scoring system in predicting postoperative morbidity and mortality. World J Surg 2009;33:80-5.

9. Arveen S, Jagdish S, Kadambari D. Perforated peptic ulcer in South India: An institutional perspective. World J Surg 2009;33:1600-4.

10. Montalvo-Javé EE, Corres-Sillas $\mathrm{O}$, Athié-Gutiérrez $\mathrm{C}$. Factors associated with postoperative complications and mortality in perforated peptic ulcer. Cir Cir 2011;79:141-8.

11. Goudar BV, Telkar S, Lamani YP, Shirbur SN, Ambi U, Hosalli V. Perforated peptic ulcer disease: Factors predicting the mortality and morbidity in a tertiary care centre in southern India. Internet J Surg 2010;27.

12. Chou NH, Mok KT, Chang HT, Liu SI, Tsai CC, Wang BW, et al. Risk factors of mortality in perforated peptic ulcer. Eur J Surg 2000;166:149-53.

13. Boey J, Wong J, Ong GB. A prospective study of operative risk factors in perforated duodenal ulcers. Ann Surg 1982;195:265-9.

14. Bertleff MJ, Lange JF. Perforated peptic ulcer disease: A review of history and treatment. Dig Surg 2010;27:161-9.

15. Singh R, Kumar N, Bhattacharya A, Vajifdar H. Preoperative predictors of mortality in adult patients with perforation peritonitis. Indian J Crit Care Med 2011;15:157-63.

16. Ozalp N, Zulfikaroglu B, Bilgic I, Koc M. Evaluation of risk factors for mortality in perforated peptic ulcer in Ankara Numune Teaching Hospital, Ankara, Turkey. East Afr Med J 2004;81:634-7.

17. Koç M, Yoldas O, Kiliç YA, Göçmen E, Ertan T, Dizen H, et al. Comparison and validation of scoring systems in a cohort of patients treated for perforated peptic ulcer. Langenbecks Arch Surg 2007;392:581-5.

18. Chan WH, Wong WK, Khin LW, Soo KC. Adverse operative risk factors for perforated peptic ulcer. Ann Acad Med Singapore 2000;29:164-7.

19. Bjerre CC, Holte K. Perioperative fluid therapy in perforated ulcers. Ugeskr Laeger 2009;171:1488-91.

20. LoCicero $\mathrm{J}^{{ }^{\text {rd }}}$, Nichols RL. Sepsis after gastroduodenal operations: Relationship to gastric acid, motility, and endogenous microflora. South Med J 1980;73:878-80.

21. Møller MH, Adamsen S, Wøjdemann M, Møller AM. Perforated peptic ulcer: How to improve outcome? Scand J Gastroenterol 2009;44: 15-22.

22. Rajesh V, Chandra SS, Smile SR. Risk factors predicting operative mortality in perforated peptic ulcer disease. Trop Gastroenterol 2003;24:148-50.

23. Buck DL, Vester-Andersen M, Møller MH, Danish Clinical Register of Emergency Surgery. Surgical delay is a critical determinant of survival in perforated peptic ulcer. Br J Surg 2013;100:1045-9.

24. Kujath P, Schwandner O, Bruch HP. Morbidity and mortality of perforated peptic gastroduodenal ulcer following emergency surgery. Langenbecks Arch Surg 2002;387:298-302.

25. Arici C, Mesci A, Dincer D, Dinckan A, Colak T. Analysis of risk factors predicting (affecting) mortality and morbidity of peptic ulcer perforations. Int Surg 2007;92:147-54.

How to cite this article: Prabhu VV, Prasadi SG, Gore A. Does resuscitation in perforation-operation interval modify the mortality in duodenal perforations?. Int J Med Public Health 2015;5:110-4. Source of Support: Nil, Conflict of Interest: None declared. 